\title{
454 pyrosequencing based transcriptome analysis of Zygaena filipendulae with focus on genes involved in biosynthesis of cyanogenic glucosides

\author{
Mika Zagrobelny 1,2, Karsten Scheibye-Alsing3,4, Niels Bjerg Jensen 1,2, \\ Birger Lindberg Møller ${ }^{1,2}$, Jan Gorodkin ${ }^{3,4}$ and Søren Bak*1,2,3
}

\begin{abstract}
Address: ${ }^{1}$ Plant Biochemistry Laboratory, Department of Plant Biology and Biotechnology, University of Copenhagen, 40 Thorvaldsensvej, DK1871 Frederiksberg C, Copenhagen, Denmark, ${ }^{2}$ The VKR Research Centre "Proactive Plants", University of Copenhagen, 40 Thorvaldsensvej, DK1871 Frederiksberg C, Copenhagen, Denmark, ${ }^{3}$ Section for Genetics and Bioinformatics (IBHV), Faculty of Life Sciences (LIFE), University of Copenhagen, 3 Grønnegårdsvej, DK-1871 Frederiksberg C, Denmark and ${ }^{4}$ Center for Applied Bioinformatics, University of Copenhagen, 40 Thorvaldsensvej, DK-1871 Frederiksberg C, Copenhagen, Denmark
\end{abstract}

Email: Mika Zagrobelny - miz@life.ku.dk; Karsten Scheibye-Alsing - scheibye@life.ku.dk; Niels Bjerg Jensen - nbj@life.ku.dk; Birger Lindberg Møller - blm@life.ku.dk; Jan Gorodkin - gorodkin@life.ku.dk; Søren Bak* - bak@life.ku.dk

* Corresponding author

BMC Genomics 2009, 10:574 doi:10.1 186/147|-2164-10-574

This article is available from: http://www.biomedcentral.com/I47I-2/64/I0/574

(c) 2009 Zagrobelny et al; licensee BioMed Central Ltd.

This is an Open Access article distributed under the terms of the Creative Commons Attribution License (http://creativecommons.org/licenses/by/2.0), which permits unrestricted use, distribution, and reproduction in any medium, provided the original work is properly cited.

\begin{abstract}
Background: An essential driving component in the co-evolution of plants and insects is the ability to produce and handle bioactive compounds. Plants produce bioactive natural products for defense, but some insects detoxify and/or sequester the compounds, opening up for new niches with fewer competitors. To study the molecular mechanism behind the co-adaption in plant-insect interactions, we have investigated the interactions between Lotus corniculatus and Zygaena filipendulae. They both contain cyanogenic glucosides which liberate toxic hydrogen cyanide upon breakdown. Moths belonging to the Zygaena family are the only insects known, able to carry out both de novo biosynthesis and sequestration of the same cyanogenic glucosides as those from their feed plants. The biosynthetic pathway for cyanogenic glucoside biosynthesis in Z. filipendulae proceeds using the same intermediates as in the well known pathway from plants, but none of the enzymes responsible have been identified. A genomics strategy founded on 454 pyrosequencing of the $Z$. filipendulae transcriptome was undertaken to identify some of these enzymes in Z. filipendulae.
\end{abstract}

Results: Comparisons of the $Z$. filipendulae transcriptome with the sequenced genomes of Bombyx mori, Drosophila melanogaster, Tribolium castaneum, Apis mellifera and Anopheles gambiae indicate a high coverage of the Z. filipendulae transcriptome. II\% of the Z. filipendulae transcriptome sequences were assigned to Gene Ontology categories. Candidate genes for enzymes functioning in the biosynthesis of cyanogenic glucosides (cytochrome P450 and family I glycosyltransferases) were identified based on sequence length, number of copies and presence/ absence of close homologs in D. melanogaster, B. mori and the cyanogenic butterfly Heliconius. Examination of biased codon usage, GC content and selection on gene candidates support the notion of cyanogenesis as an "old" trait within Ditrysia, as well as its origins being convergent between plants and insects.

Conclusion: Pyrosequencing is an attractive approach to gain access to genes in the biosynthesis of bio-active natural products from insects and other organisms, for which the genome sequence is not known. Based on analysis of the $Z$. filipendulae transcriptome, promising gene candidates for biosynthesis of cyanogenic glucosides was identified, and the suitability of $Z$. filipendulae as a model system for cyanogenesis in insects is evident. 


\section{Background}

The advent of new sequencing technologies opens up the opportunity to engage in genomics in non-model organisms, and to study model systems aimed at specific interactions in a biological context. In this paper, we have taken a genomics approach to unravel co-adaptation within a plant-insect interaction at the molecular level, investigating the refined metabolic interactions between the six-spot burnet moth, Zygaena filipendulae, and its host plant bird's-foot trefoil, Lotus corniculatus.

Plants and insects have co-evolved for the last 400 million years. A key driving component in these interactions is the ability to produce and handle bioactive natural products. The dogma is that plants produce low molecular mass bioactive compounds as a chemical defense against herbivorous insects. These specialized bioactive compounds are often specific to a particular species. As a countermeasure, herbivorous insects evolve strategies to circumvent the plants chemical defense systems, e.g. by detoxification. The ability to circumvent the chemical defense system of a particular plant species enables the insect to develop an advantageous niche with few competitors. This has resulted in the majority of insects having a restricted diet, feeding only on one plant species.

In some cases, insects are able to de novo biosynthesize the same or similar defense compounds as the feed plant, which is why they are immediately able to handle the defense compounds when they encounter the plant. These insects use the compounds in their own defense against predators. If the insects develop an ability to sequester the particular bio-active natural product from the feed plants, the interaction may become highly beneficial for the insects, as they save energy in their own metabolism.
To unravel the molecular mechanism behind co-adaption and balance between sequestration and de novo biosynthesis of bioactive compounds in plant-insect interactions, we have taken advantage of the model system of interactions between $L$. corniculatus and $Z$. filipendulae [1]. L. corniculatus and $Z$. filipendulae both contain the bioactive compounds cyanogenic glucosides (CNglcs) which can liberate toxic hydrogen cyanide by hydrolysis. While CNglcs are widespread in plants [2], they are in general rare in arthropods [3]. Within insects, particular within butterflies and moths, several species are known to sequester or de novo biosynthesize CNglcs $[1,4,5]$. Moths belonging to the Zygaena family are the only insects known, able to de novo biosynthesize as well as to sequester the same CNglcs $[1,5]$.

In plants, the entire pathway for CNglc biosynthesis has been elucidated [6-10]. The precursors for CNglcs are amino acids, and the biosynthetic pathway is genetically relatively simple as only two multifunctional cytochromes P450 (P450) and a family 1 glycosyltransferase (UGT) are involved (Figure 1). In contrast, knowledge on biosynthesis of CNglcs in insects is rudimentary. It has been shown that Zygaena trifolii is able to biosynthesize the CNglcs linamarin and lotaustralin using intermediates equivalent to those identified in plants [11-13], and preliminary evidence show that the pathway in $Z$. filipendulae also involves P450 enzymes [14] (Figure 1). It is not known whether the ability to biosynthesize CNglcs in insects is due to horizontal gene transfers, divergence, or convergence.

To identify gene candidates involved in de novo biosynthesis of CNglcs in Z. filipendulae 454 pyrosequencing of the transcriptome was carried out. The transcriptome was compared to the deduced coding sequences of five known

\section{Biosynthesis of lotaustralin and linamarin}

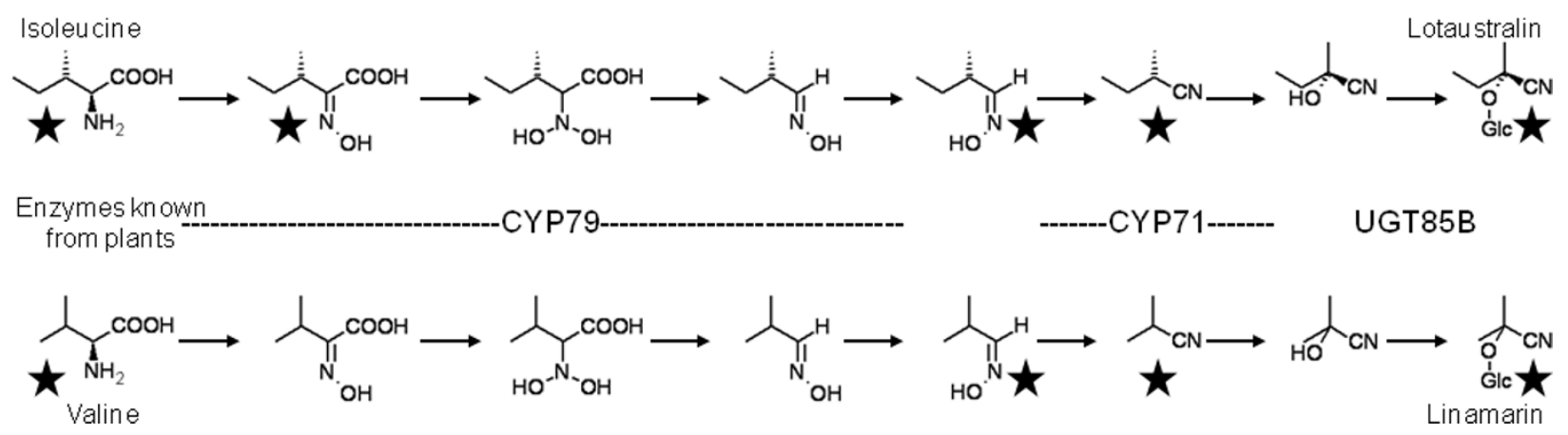

\section{Figure I}

Proposed biosynthetic pathway for the CNglcs linamarin and lotaustralin. The types of enzymes catalyzing the equivalent reactions in plants are shown. Intermediates known from insects are indicated with a star. 
insect genomes: Bombyx mori, Drosophila melanogaster, Tribolium castaneum, Apis mellifera, and Anopheles gambiae. As P450s and UGTs are key enzyme families involved in secondary metabolism in plants as well as in insects [15], a focused analysis of these two multigene families were conducted, to specify gene candidates involved in CNglc biosynthesis.

\section{Results}

\section{Genome annotation}

454 pyrosequencing was carried out on cDNA from a $Z$. filipendulae larva feeding on leaves of acyanogenic $L$. corniculatus. Larvae reared on acyanogenic L. corniculatus were previously shown to accumulate the same overall amount of CNglcs as larvae reared on cyanogenic $L$. corniculatus [16]. This balance is achieved by de novo biosynthesis of the CNglcs, and imply that the transcript levels for the biosynthetic enzymes are elevated [16]. To achieve depth in the transcriptome by decreasing the number of reads for highly expressed genes, the cDNA library was normalized prior to sequencing. Pyrosequencing resulted in 319,956 reads [NCBI Short Read Archive, accession SRX008323] which were assembled into 29,857 contigs and 42,215 singlets. The average length of reads is 229 nucleotides, while the average contig length is 329 nucleotides, supported on average by 4.4 reads. 3,938 contigs were longer than 500 bp (Figure 2).

The Z. filipendulae transcriptome was GO-annotated based on matches to uniprot proteins. The degree of confidence to the assignment of contig or singlet to a particular GOannotated protein from $\mathrm{nr}$ and Uniprot databases is presented as match levels in Table 1[17]. For example, M2 signifies a requirement of the contig to have an identity of at least $85 \%$ measured within the matching region, and to cover at least $90 \%$ of the matching database sequence. On the basis of a match level 3 (70\% identity/70\% coverage) or better match, 158 contigs were assigned to a uniprot protein with associated GO-terms, while 335 contigs were associated with GO-terms at match level 4 (60\% identity/ $50 \%$ coverage) or better. Upon relaxing the match level criteria to level 5 (20\% identity/20\%coverage) or better, 3,240 contigs could be associated with one or more GOterms ( $\sim 11 \%$ of the $Z$. filipendulae transcriptome). Because several of the sequences were characterized by more than one GO term, the total number of GO terms obtained at match level 5 was 8,865. Most of the GO matches based on comparisons at match level 4 and better are obtained with D. melanogaster proteins (34\%), while at match level 5 and better most matches are obtained with human proteins (41\%) and only $17 \%$ with proteins from D. melanogaster. This is probably due to the much greater number of GO annotated human genes as compared to insect genes.

To group the proteins with associated GO terms, the top level term for each GO category "Molecular function", "Biological Process" and "Cellular component" was recorded at the different match levels. The distribution of hits in the different categories turned out to be roughly similar for each match level. The distribution of terms for match level 5 or better is shown in Figure 3, and a table listing the different terms and their respective counts for each match level is provided in Additional file 1. The dominant terms for "Molecular function" are clearly transporter activity and binding, while the dominant term for "Biological process" is pigmentation. Within "Cellular
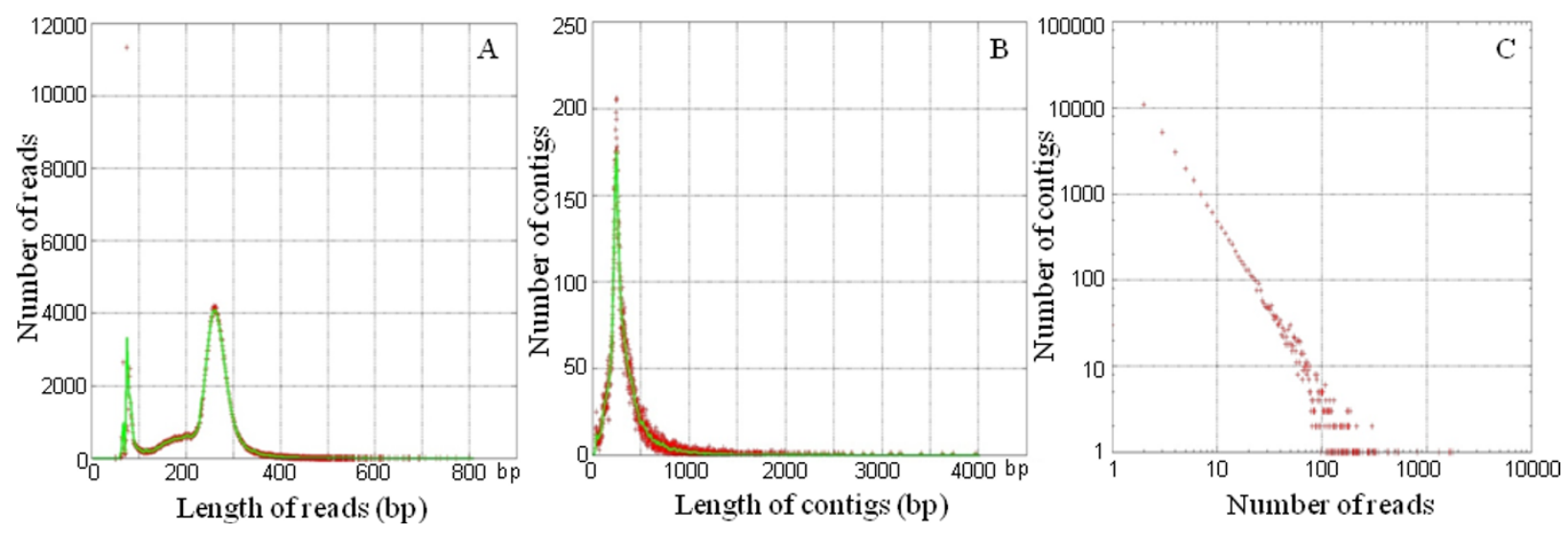

Figure 2

Distribution of sequence lengths and cluster sizes. A: Number distribution of lengths of the individual reads. B: Number distribution of lengths of the contigs. Average contig length is indicated by a green line. C: Contig sizes. Contig sizes are scaling invariant as observed by [I7]. 
Table I: Protein BLAST statistics

\begin{tabular}{|c|c|c|c|c|}
\hline Match level (identity/coverage) & nr (contigs) & nr (singlets) & Uniprot (contigs) & Uniprot (singlets) \\
\hline M0 (98\%/ / 00\%) & 33 & 0 & 21 & 0 \\
\hline MI (95\%/95\%) & 58 & 2 & 31 & 0 \\
\hline M2 (85\%/90\%) & 142 & 5 & 49 & 3 \\
\hline M3 (70\%/70\%) & 495 & 70 & 163 & 16 \\
\hline M4 (60\%/50\%) & 1019 & 200 & 326 & 64 \\
\hline M5 (20\%/20\%) & 6466 & 2744 & 4685 & 1276 \\
\hline
\end{tabular}

Number of contigs and singlets in each match level that match proteins in $\mathrm{nr}$ and Uniprot databases. For example $\mathrm{M} 2$ means a requirement of the contig to have an identity of at least $85 \%$ measured within the matching region and to cover at least $90 \%$ of the matching database sequence.

component" the dominant terms are evenly divided between organelle, cell part and organelle part.

\section{Genome comparison}

Comparisons of the translated $Z$. filipendulae transcriptome to B. mori, D. melanogaster, A. mellifera, A. gambiae, and $T$. castaneum protein sequences using BLASTx are shown on Figure 4 . The average identity of the protein sequence matches between $B$. mori and $Z$. filipendulae is greater than between $Z$. filipendulae and D. melanogaster,
A. gambiae, A. mellifera, and T. castaneum (Figure 4). This is to be expected, as phylogenetically B. mori is closer to $Z$. filipendulae (both belong to ditrysia) than the other species are (belonging to Diptera, Hymenoptera and Coleoptera) (Figure 5A). D. melanogaster, T. castaneum, and A. mellifera have intermediate identities of BLAST hits matching their evolutionary distance from $Z$. filipendulae, whereas A. gambiae seem to have more divergent sequences (Figure 4). This could be caused by adaptation of A. gambiae to blood feeding and development of insec-
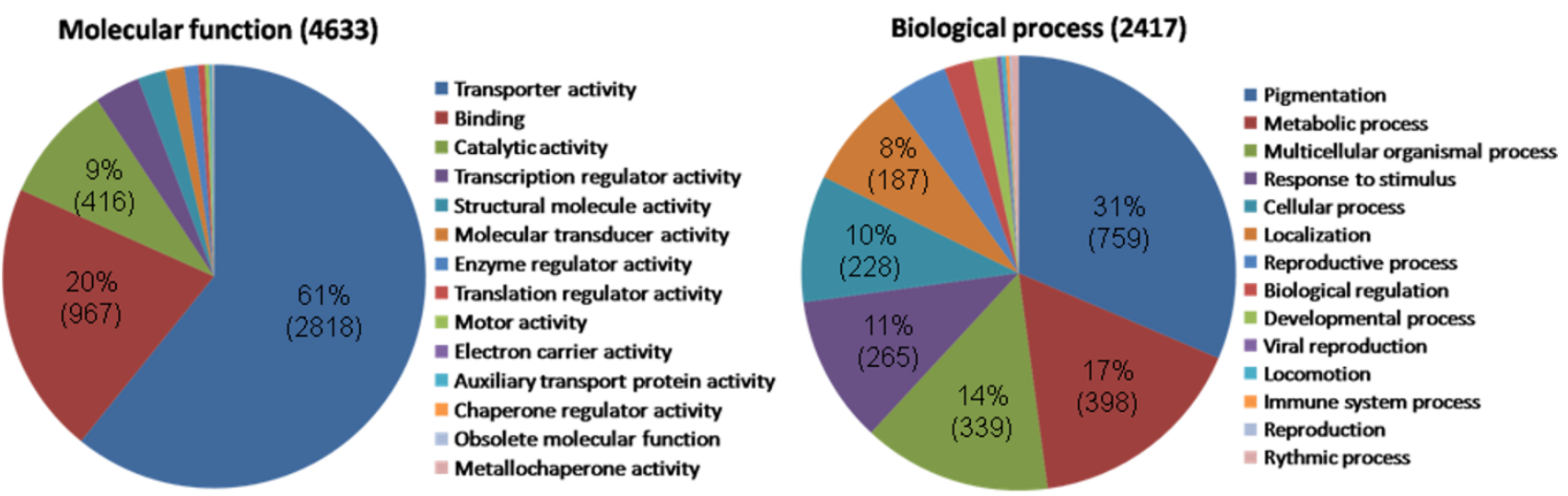

Cellular component (1815)

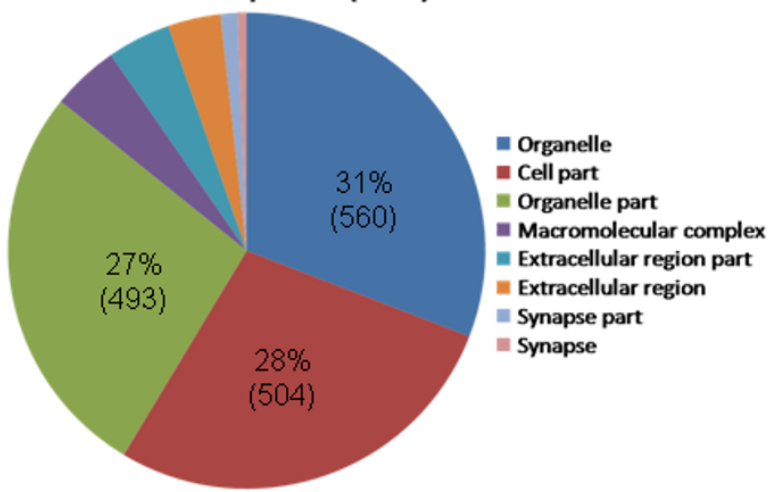

Figure 3

Gene ontology (GO) -terms for Z. filipendulae proteins belonging to match level 5. The percentage, (total number), and distribution of top-level GO-terms for match level 5 and better in the categories "Molecular function", "Biological Process" and "Cellular component". Total number of $Z$. filipendulae genes in each category is also shown. 


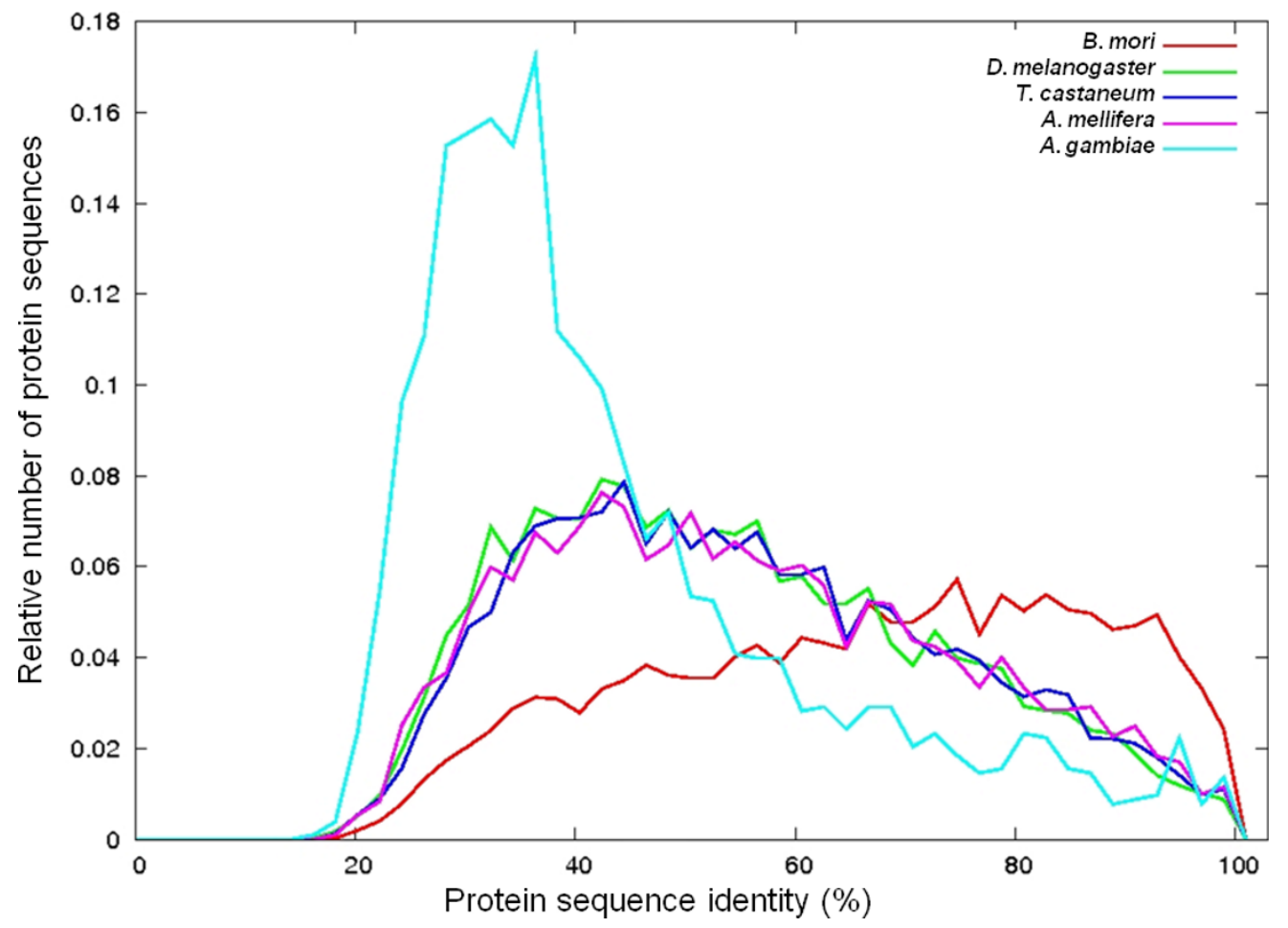

\section{Figure 4}

A comparison of the distribution of sequence identity of $Z$. filipendulae proteins to proteins from five different insect species. Numerical distribution of protein sequence identity between $Z$. filipendulae protein sequences obtained, and the best BLASTp search hit when compared to available B. mori, D. melanogaster, T. castaneum, A. mellifera, and A. gambiae protein sequences. All curves are normalized so that the area beneath each curve sums to I. The drop in matches with less than roughly $25 \%$ identity is probably an artifact of the BLAST E-value threshold.

ticide resistance [18], as well as a generally faster divergence in Diptera $[19,20]$. For sequences with $90-100 \%$ identity, numbers are very similar for $D$. melanogaster, $T$. castaneum, A. mellifera, and A. gambiae, probably reflecting housekeeping genes (Figure 4). The BLASTx searches of the $Z$. filipendulae sequences against the $D$. melanogaster protein dataset, resulted in 8,128 contigs and 4,780 singlets matching 11,365 and 8,693 D. melanogaster proteins, respectively, with a cut-off E-value of $1 e^{-3}$. The BLASTx search of the $Z$. filipendulae sequences against the B. mori consensus protein dataset resulted in 10,824 contigs and 7,513 singlets matching 8,195 and $6,993 \mathrm{~B}$. mori proteins respectively. Due to overlap, the matches correspond to a total of 9,760 different $B$. mori proteins. The larger number of BLASTx hits between D. melanogaster and $Z$. filipendulae compared to the number of hits between $B$. mori and $Z$. filipendulae probably reflects the greater number of recorded $D$. melanogaster proteins compared to B. mori proteins $(19,389$ versus 14,623$)$. Figure 5B presents a Venn diagram of the matches from contigs (upper) and singlets (lower) belonging to the Z filipendulae transcriptome compared to the D. melanogaster and $B$. mori protein datasets, respectively (Figure 5). The num- bers 18,580 and 34,686 denote the number of contigs and singlets, respectively, from Z filipendulae without matches to either $D$. melanogaster or $B$. mori. The high number of $Z$. filipendulae sequences which do not match either $D$. melanogaster or B. mori (Figure 5) probably reflects a mixture of novel, unique genes, poorly conserved genes, and erroneous sequences.

Heliconius butterfly species are known to biosynthesize linamarin and often lotaustralin [21,22], and are more closely related to Zygaenidae than $D$. melanogaster and $B$. mori (Figure 5). This prompted a sequence comparison between $Z$. filipendulae and three Heliconius species. The Heliconius species represented in the sequence databases are H. melpomene $(4,977 \mathrm{ESTs})$ and H. erato $(17,567 \mathrm{ESTs})$, with the H. erato data set containing additional ESTs from the closely related species $H$. himera [23]. These two data sets were assembled separately using the EST sequence assembler geneDistiller (Scheibye-Alsing $\mathrm{K}$ et al: EST assembly with genedistiller, submitted), resulting in, respectively, 420 and 1,207 contigs and 1,205 and 4,438 singlets. In total, 3,961 Z. filipendulae contigs had detectable similarity (E-value $1 \mathrm{e}^{-3}$ ) with sequences in the Helico- 


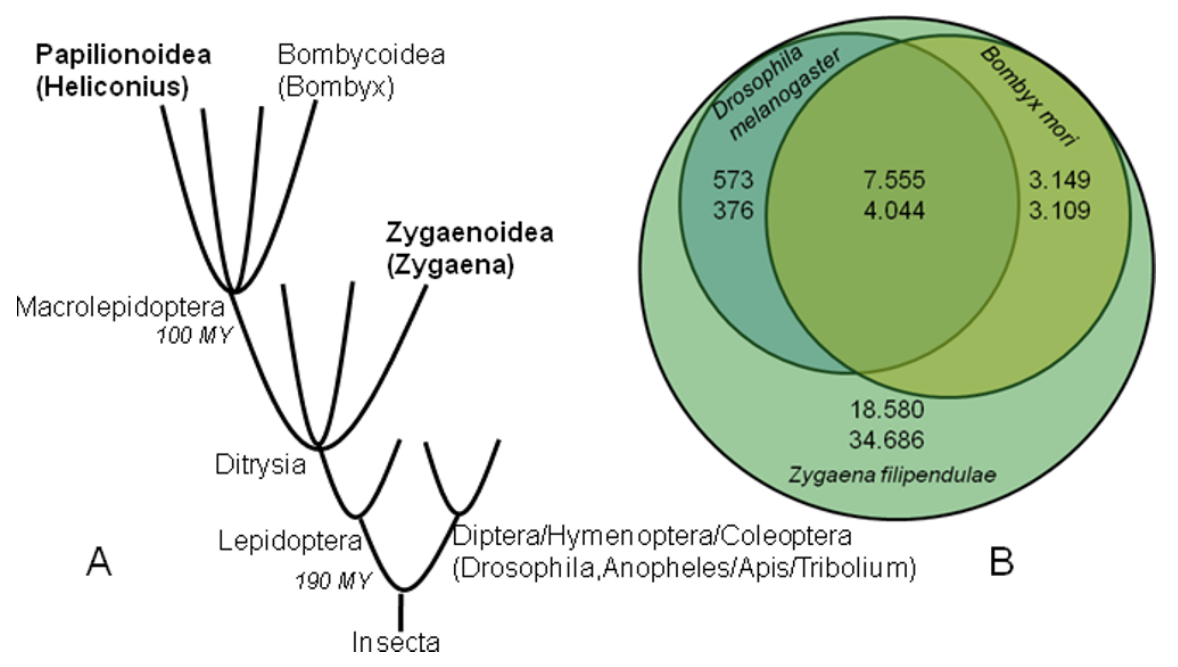

Figure 5

Phylogenetic relationships and genetic overlap between Z. filipendulae, B. mori and D. melanogaster. A: Simplified evolutionary tree showing the relationships between Zygaena, Heliconius, Bombyx, Drosophila, Anopheles, Apis, and Tribolium. Groups comprising cyanogenic species are shown in bold. Time since divergence of the lineages Papilionoidea/Bombycoidea and Lepidoptera/Diptera are shown in italics [20]. B: Venn diagram of the number of contigs (upper number) and singlets (lower number) from Z. filipendulae which show matches to D. melanogaster (19,389 proteins) and/or B. mori (I4,623 proteins). Cut-off E-value was $\mathrm{Ie}^{-3}$.

nius data sets (BLASTn), where 1,626 matched Heliconius contigs and 2,723 matched Heliconius singlets. In addition, 3,138 singlets from $Z$. filipendulae matched Heliconius sequences, with 1,117 singlets matching Heliconius contigs and 2,345 matching Heliconius singlets. In total, 4,035 of the 7,270 conreads (contigs and singlets) of the Heliconius datasets could be associated with $Z$. filipendulae (E-value $\left.1 \mathrm{e}^{-3}\right)$, corresponding to $55 \%$ of the Heliconius conreads. On the other hand, only 7,099 of the 72,072 conreads from $Z$. filipendulae, corresponding to $9.8 \%$, are represented by Heliconius conreads, which most likely reflects the more extensive sampling of the $Z$. filipendulae transcriptome. Additionally, the fact that the 7,099 Z. filipendulae sequences hit only 4,035 different Heliconius conreads implies that there is a degree of under clustering in the $Z$. filipendulae dataset, i.e. some reads representing the same gene have not been clustered together in the same contig. This was also experienced in the course of manual merging different $Z$. filipendulae contigs within gene families (see Methods). In addition, many of the Heliconius ESTs originate from wing disc cDNA libraries [24] which, unfortunately, may not have a high expression of genes involved in $\mathrm{CNglc}$ biosynthesis.

We used BLASTx for the comparisons of $Z$. filipendulae to $D$. melanogaster and B. mori because good BLAST hits are more easily obtained with amino acid sequences than with nucleotide sequences, if sequences have diverged much from each other. Because the Heliconius dataset consists of nucleotide sequences, BLASTn had to be used for comparisons between $Z$. filipendulae and Heliconius. Since it was not directly possible to compare the BLASTx searches to the BLASTn searches, test BLASTn runs were performed between $Z$. filipendulae sequences and the genomes of $B$. mori and D. melanogaster. These runs showed that for $D$. melanogaster and to a lesser degree $B$. mori, sequences were so diverged that the number of valid matches obtained was limited. However, using rough estimates we find that around 39\% of the Heliconius EST sequences show similarity with $B$. mori.

\section{Candidates for enzymes involved in biosynthesis of cyanogenic glucosides}

Our working hypothesis is that the same gene families (P450s and UGTs) are involved in biosynthesis of CNglcs in plants and insects (Figure 1). On account of that, a general search for P450s and UGTs with sequences from both plants [25] and insects was carried out in the $Z$. filipendulae transcriptome. Despite normalization of the cDNA library, transcripts representing enzymes involved in CNglc biosynthesis would be expected to be abundant, because the pathway for $\mathrm{CNglc}$ biosynthesis was induced by rearing the $Z$. filipendulae larvae on acyanogenic $L$. corniculatus leaves. Full length or almost full length contigs supported by a high number of pyrosequencing reads would therefore be expected lead candidates for enzymes involved in CNglc biosynthesis. A second important selection criterion is genes which do not have a close homolog in B. mori and/or D. melanogaster, because these insects are not biosynthesizing CNglcs. 


\section{Cytochromes P450 (P450)}

P450s are heme-thiolate proteins present in all kingdoms involved in primary and secondary metabolism as well as in detoxification [2]. Particularly in plants, they are often recruited for novel biosynthetic pathways, and in plants the first two steps in $\mathrm{CNglc}$ biosynthesis are catalyzed by P450s. In insects, P450s play crucial roles in the detoxification of toxic bio-active plant natural products present in their feed plants, as well as in primary metabolism and development. In general, insect genomes harbor $\sim 100$ different P450s, which can be divided into four clades http:/ /p450.sophia.inra.fr/[26,27]. For the majority of P450s from plants as well as insects the biochemical function is unknown. However, some functionality can be assigned to each of the four clades based on known functionalities. The CYP6, CYP9, CYP28, CYP308-310, and CYP321 families constitute the first clade, and many of the genes belonging to these families are known to mediate responses to environmental challenges. The families are characterized by a very high diversity, individual members have proliferated by duplication events, show rapid rates of evolution, occur in gene clusters, and show tissue- or developmental-specific expression. The CYP4, CYP311313, CYP316, and CYP325 families belong to the second clade of highly diverse insect P450s, with some of the gene members being induced by xenobiotics. The third clade is the mitochondrial CYP12 family, comprising many rapidly evolving genes. Some of the slowly evolving genes in this family are involved in moulting. The CYP15, CYP18, and CYP303-307 families constitute the fourth clade of insect P450s. Some members of these families are involved in essential physiological functions like juvenile hormone biosynthesis and moulting.

Approximately 120 putative P450 conreads were identified in the $Z$. filipendulae transcriptome (a table of all sequences with length and coverage are shown in Additional file 1). Five $Z$. filipendulae $\mathrm{P} 450$ sequences (ZfCYP9A37, ZfCYP304F2, ZfCYP332A3, ZfCYP379A2, ZfCYP379A3) were identified in the dataset as full length sequences. The coding sequence of a P450 is approximately 1500 bp. Eleven P450 sequences longer than 1000 nucleotides were discovered all in all (ZfCYP4G47, ZfCYP4G48, ZfCYP9A37, ZfCYP304F2, ZfCYP332A3, ZfCYP333B8, ZfCYP379A2, ZfCYP379A3, ZfP450-5, ZfP450-13, Zfc3346). To extend these, and other partial sequences, RACE PCR was performed, and this provided further full length sequences of ZfCYP4G47, ZfCYP4G48, ZfCYP4L17, ZfCYP6CT1, ZfCYP6AE27, CYP9A36, and ZfCYP333B8, resulting in twelve full length P450 sequences [GenBank accessions GQ915312-GQ915323]. A Neighbor-Joining tree containing the twelve full length $Z$. filipendulae sequences as well as selected P450s from other species is shown in Figure 6. The selected P450s were most closely related to the $Z$. filipendulae P450s, and

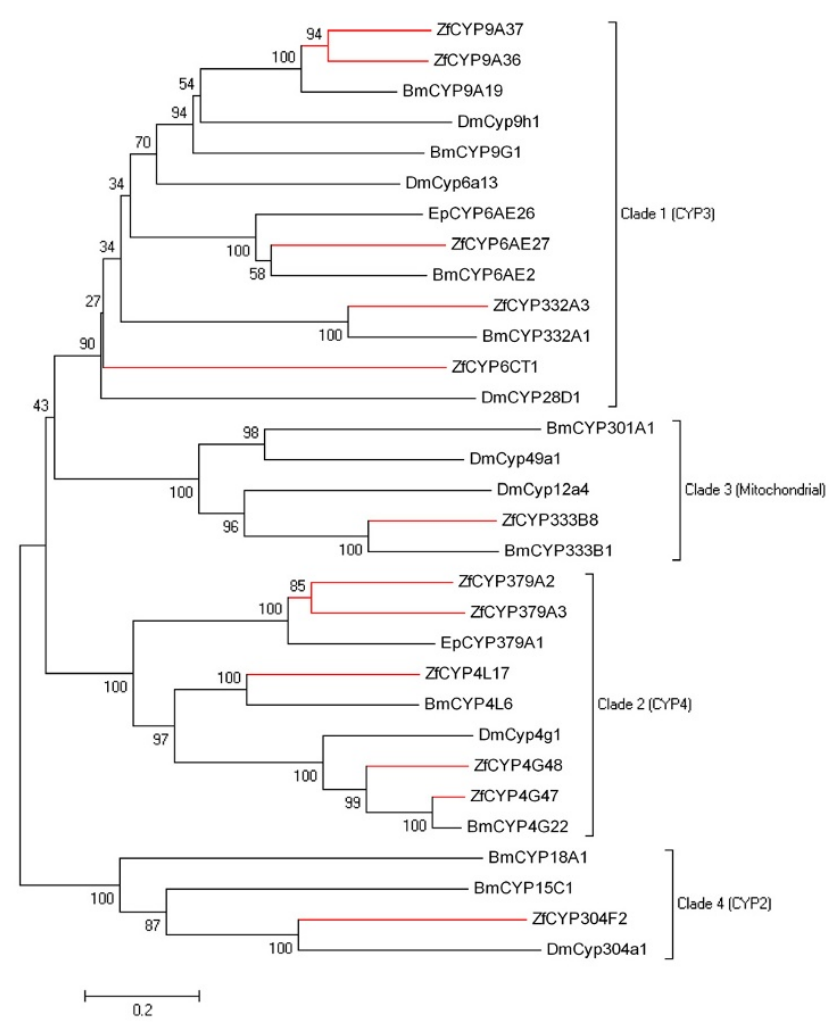

Figure 6

Neighbor-Joining bootstrap tree of translated full length Z. filipendulae P450 sequences. Sequences from $Z$. filipendulae are marked in red. Clades of insect P450s are according to [26,27]. Bm: B. mori, Dm: D. melanogaster, EP: E. postvittana. Bootstrap values are shown as percentages.

were chosen from a much larger tree (Additional file 2) comprising more than 200 P450 genes from many different insect species.

The few P450 sequences from the tree with assigned functions are: DmCYP4G1, DmCYP49A1, DmCYP12A4, and BmCYP15C1. DmCYP4G1 is required for lipid metabolism, DmCYP49A1 and DmCYP12A4 confer insecticide resistance and may therefore be involved in detoxification reactions, while BmCYP15C1 is involved in juvenile hormone biosynthesis. For an overview of known D. melanogaster P450 functions see [28]. Based on the known function and its position in the tree, it can be inferred that ZfCYP304F2 may be involved in juvenile hormone biosynthesis, ZfCYP333B8 may be involved in detoxification processes, and ZfCYP4G48 and ZfCYP4G47 could be required for lipid metabolism. However, because the $Z$. filipendulae P450 sequences are not that closely related to the P450s with known functions, these functional assignments of P450s in Z. filipendulae are to be considered tentative. Because most of the P450s from B. mori and D. melanogaster shown in the tree has not been properly 
characterized, it was not possible to infer functions to most of the full length Z. filipendulae P450s obtained.

Based on our criteria for lead candidates, ZfCYP4G47 would appear to be a less likely candidate, because of the close homolog present in $B$. mori ( $82 \%$ sequence identity on the amino acid level). ZfCYP4G47 was the only one of the $Z$. filipendulae P450s with a close homolog in the cyanogenic butterfly Heliconius, showing $83 \%$ sequence identity over 250 amino acid residues. As the sequence is partial it has been omitted from the tree presented in Figure 6, and due to the close homolog in B. mori mentioned earlier, it is not a good candidate for $\mathrm{CNglc}$ biosynthesis. The remaining eleven full length $Z$. filipendulae P450s appear sufficiently distant to genes in the other two species, to have been able to acquire functions specific to $Z$. filipendulae. The most divergent $Z$. filipendulae full length P450 is ZfCYP6CT1 ( $30 \%$ sequence identity on the amino acid level). This may therefore be the best candidate for a P450 involved in the CNglc pathway. The Z. filipendulae transcriptome contains two P450s belonging to a new animal P450 family (CYP379 in clade 2) to which a single sequence from Epiphyas postvittana (light brown apple moth) has previously been assigned (Nelson, D. personal communication). Like Z. filipendulae, E. postvittana belong to Ditrysia (Figure 4). Since E. postvittana is not cyanogenic, the two putative CYP379 orthologs are not obvious candidates for a P450 involved in CNglc biosynthesis.

\section{UDPG-Glucosyltransferases (UGTs)}

The third and final step in CNglc biosynthesis in plants is a glucosylation step catalyzed by a Family 1 UDPG-glycosyltransferase [29], and mediating the conversion of a cyanohydrin into the corresponding CNglc [30,31]. Family 1 UGTs are ubiquitously found in plants, animals, fungi, bacteria, and viruses [32]. UGTs generally serve to glycosylate low molecular weight molecules, as a mean to convert reactive and toxic aglycones into more stable and non-reactive storage forms, as well as to increase solubility of hydrophobic metabolites, and as transport cues for intra- and intercellular transport [32]. In addition, UGTs are also involved in hormone homeostasis and detoxification of xenobiotics.

The pyrosequencing approach resulted in identification of 41 different putative Family 1 UGT conreads in the Z. filipendulae transcriptome. Three of these sequences represented full length sequences (ZfUGT33A1, ZfUGT33B1, ZfUGT35E1) [GenBank accessions GQ915324GQ915326] (Figure 7) (a table of all sequences with length and coverage are presented in Additional file 1). A Neighbor-Joining tree with full length $Z$. filipendulae UGTs as well as UGTs from other insects is shown in Figure 7. The genes most closely related to the $Z$. filipendulae UGTs

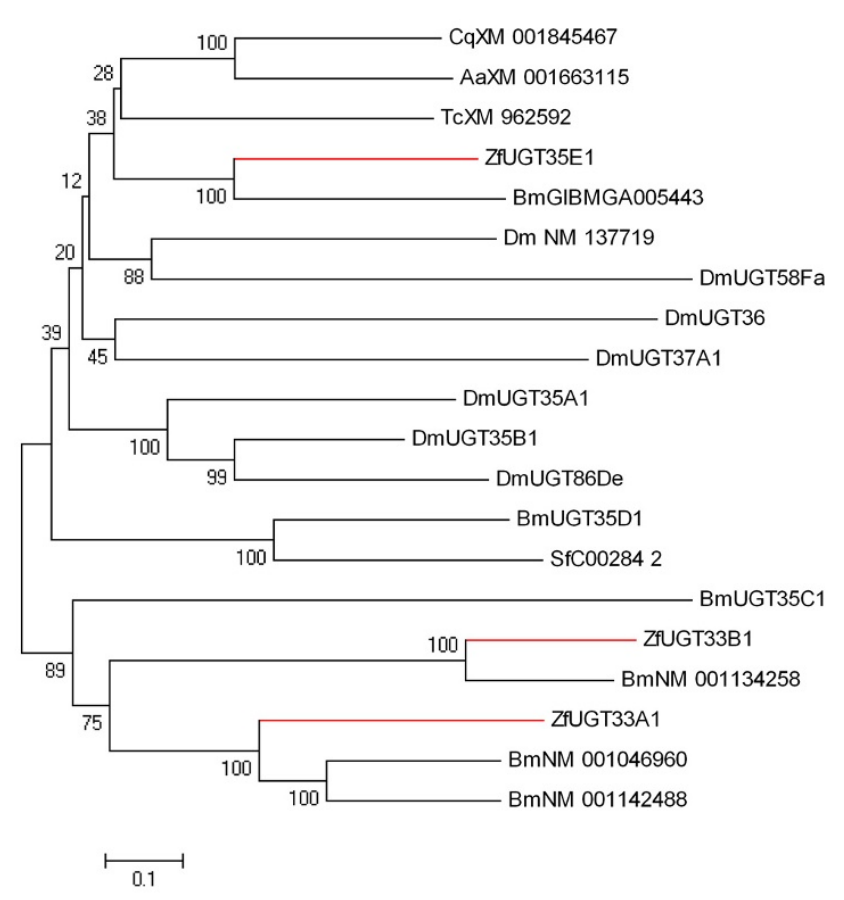

Figure 7

Neighbor-Joining bootstrap tree of translated full length $Z$. filipendulae UGT sequences. Sequences from $Z$. filipendulae are marked in red. Aa:Aedes aegypti, Bm:B. mori, Cq:Culex quinquefasciatus, Sf:S. frugiperda, Tc:T. castaneum.

Bootstrap values are shown as percentages.

were selected from a larger tree (Additional file 3) comprising 76 UGT genes from different insect species.

The high abundance of the three full length $Z$. filipendulae UGT transcripts implies that one of these three UGT sequences may be the UGT catalyzing the last step in $\mathrm{CNglc}$ biosynthesis. From Figure 7 it is evident that the $Z$. filipendulae UGTs assigned as ZfUGT33A1 and ZfUGT33B1 have fairly close homologs in B. mori, while this is not the case for ZfUGT35E1. Accordingly, among the full length sequences obtained, ZfUGT35E1 would therefore be a lead candidate.

\section{Codon usage and GC content}

Genes involved in biosynthesis of CNglcs in Z. filipendulae have been hypothesized to be highly expressed in larvae feeding on acyanogenic L. corniculatus [16]. Highly expressed genes are generally assumed to possess a more biased codon usage, and a higher GC content in comparison to genes with low expression [33]. Accordingly, to obtain evidence of their expression levels, codon usage and GC content of each Z filipendulae candidate gene encoding P450s or UGTs were examined. Codon usage and GC content of genes encoding P450s and UGTs were similar, and average numbers for each gene family are 
shown in Table 2. Overall codon usage in Z. filipendulae was found to be similar to codon usage in B. mori (Table 2). A biased codon usage (ENC) is a measure of how far the codon usage of a gene departs from equal usage of synonymous codons, and is rated from 20 (maximally bias) to 61 (unbiased) [33]. The high ENC numbers signify a low codon usage bias, i.e. a somewhat equal usage of synonymous codons within the gene families. D. melanogaster has the lowest ENC values, e.g. higher codon usage bias compared to $Z$. filipendulae and B. mori. Codon usage bias within the P450 genes is significantly different when $D$. melanogaster sequences are compared to sequences from $Z$. filipendulae and B. mori using student t-tests (both significant at the $99 \%$ level). This was also the case when only 12 D. melanogaster P450s were compared to the $12 \mathrm{Z}$. filipendulae P450s (data not shown). Codon usage within the UGTs showed no significant differences between the three species. The high number of $\mathrm{P} 450$ genes available for analysis in comparison to the number of UGT genes available, may explain the insignificant codon usage differences observed in the latter group. It has previously been suggested that Lepidoptera exhibits less codon usage bias than other species [34], and this study corroborates with that observation.

The GC content in the $Z$. filipendulae transcriptome is $38.7 \%$ (Table 2). This is lower than measured for A. gambiae $(55.8 \%)$, D. melanogaster $(53.9 \%)$, B. mori $(48.1 \%)$, and T. castaneum $(47.2 \%)$, but more similar to the GC content in A. mellifera (43.5\%) and Heliconius (44\%). This is remarkable, because $Z$. filipendulae and Heliconius are phylogenetically more closely related to $B$. mori and $D$. melanogaster [35], in comparison to the relationship between $Z$. filipendulae and A. mellifera. For unknown reasons, selective pressure within Ditrysia (Figure 4) towards the development of a lower GC content may have occurred. Alternatively, the unexpected high values of GC content may have been obtained due to a prediction bias towards more GC rich transcripts, introduced by the gene annotation methods when applied on entire genomes [36].

\section{Substitution rate ratios}

To test our lead candidates for selection, we calculated the nonsynonymous $\left(d_{N}\right)$ to synonymous $\left(d_{S}\right)$ substitution rate ratio $\left(\omega=d_{N} / d_{S}\right)$ on full length sequences of P450s and UGTs from $Z$. filipendulae. $\omega$ provides a sensitive measure of selective pressure at the protein level, with $\omega$ values $<1,=1$ and $>1$ indicating purifying selection, neutral evolution and diversifying selection, respectively. Maximum likelihood models of codon substitution account for variable selective pressures among amino acid sites. By testing different models with more classes of $\omega$, positive selection in specific regions of a gene can be detected [37]. The program codeml from the PAML4.1 package was used for the analysis. Site-models $0,1,2,3$, $5,7,8$ from codeml were tested on the available full length P450 and UGT sequences with likelihood ratio tests [38]. The models used basically implement different rates and classes of $\omega$ values, e.g. model 0 has one $\omega$ value calculated from the data set, model 1 has two $\omega$ values, with one value fixed at 1 and the other calculated from the data set, and so on with model 8 operating with 11 classes of $\omega$ values all calculated from the data set. The likelihood ratio test was used to calculate which of the possible models offered the best fit to the data set [37]. If positive selection was present in one or more of the sequences tested, residues with positive selection would be identified in the output file.

In our analyses, average $\omega$ values range from 0.18 to 0.53 , with UGTs having the highest $\omega$ value (Table 3 ). These $\omega$ values are quite low and signify purifying selection. Model 3 (three discrete classes of $\omega$ values) provides the best fit for P450s while model 1 (two $\omega$ values of which one is fixed at 1) offers the best fit for UGTs. This demonstrates a negative purifying selection to maintain the sequence in both gene families. Negative purifying selection pressure is less restrained in some sequence domain areas for

Table 2: GC content of the transcriptome and within P450s and UGTs in Z. filipendulae, B. mori, D. melanogaster, and Heliconius species.

\begin{tabular}{|c|c|c|c|c|c|c|c|c|c|c|}
\hline & \multicolumn{3}{|c|}{ Z. filipendulae } & \multicolumn{3}{|c|}{ B. mori } & \multicolumn{3}{|c|}{ D. melanogaster } & \multirow[t]{2}{*}{ Heliconius species } \\
\hline & GC & ENC & No. & GC & ENC & No. & GC & ENC & No. & \\
\hline Transcriptome & $39 \%$ & & & $48 \%$ & & & $54 \%$ & & & $43 \%$ \\
\hline P450 & $43 \%$ & 56 & 12 & $43 \%$ & 55 & 70 & $51 \%$ & 51 & 81 & \\
\hline UGT & $40 \%$ & 55 & 3 & $42 \%$ & 56 & 5 & $48 \%$ & 56 & 7 & \\
\hline Average of groups & $42 \%$ & 56 & 15 & $43 \%$ & 56 & 75 & $50 \%$ & 54 & 88 & \\
\hline
\end{tabular}

The number of sequences used for the individual analyses are shown in column labeled "No.". Heliconius does not offer calculations on individual gene families due to the availability of too few sequences. The GC content calculated for the $D$. melanogaster, $B$. mori, and $A$. mellifera transcriptomes are based on all coding sequences deposited in Genbank, while the GC content of the $Z$. filipendulae transcriptome is based on 29.857 contigs. The GC content of the Heliconius transcriptome is derived from 1.627 contigs. 
Table 3: Nonsynonymous/synonymous substitution rate ratios $(\omega)$ in gene families from $Z$. filipendulae.

\begin{tabular}{llll}
\hline Enzymes from $Z$. filipendulae & Average $\omega\left(\boldsymbol{d}_{\boldsymbol{N}} / \boldsymbol{d}_{\boldsymbol{s}}\right)$ & Best-fit model from PAML4.I \\
\hline P450 & 0.18 & Model 3: & $\mathrm{p}: 0.030 .400 .57$ \\
UGT & 0.53 & Model I: & $0.000 .100 .25 \$$ \\
Average & 0.540 .46 & $\omega: 0.131 .00 \$$ \\
\hline
\end{tabular}

P450s as evidenced by the obtained best fit using Model 3 which is based on three discrete classes of $\omega$ values. UGTs possess larger sequence domains (46\%) where they seem to undergo neutral evolution corresponding to a $\omega$ value of 1 . This signifies that some areas are prone to accumulate a lot of mutations which could give rise to proteins with new catalytical functions or altered substrate specificities $[39,40]$. No sites with positive selection were detected in any sequence from any of the gene families (Table 3).

\section{Discussion \\ Characterization of the Zygaena filipendulae transcriptome}

The Z. filipendulae transcriptome was compared to other sequenced insect species, and the results clearly show that $Z$. filipendulae is more closely related to $B$. mori compared to D. melanogaster, A. mellifera, A. gambiae, and T. castaneum (Figure 4, Figure 5). The high percentage of $Z$. filipendulae contigs with no match in Heliconius and $B$. mori as well as in any other sequenced insects reflects the uniqueness of the species, and corroborates with previous observations of unusually many unique genes in the other Ditrysians B. mori and Manduca sexta [41]. GO groups could be assigned to $11 \%$ of the $Z$. filipendulae sequences whereas the corresponding number for the Heliconius sequences was 25\% [24]. The gene sequences encoding the gene families of relevance to cyanogenesis (P450s and UGTs) are not well represented among the genes to which a GO annotation was assigned. For the P450s, only 45 out of 118 sequences (38\%) had a BLAST hit (E-value 0.01 ) with a GO term attached. This demonstrates that the assignment of GO terms is biased towards genes with high overall sequence conservation, and well-known roles in primary metabolism.

In H. melpomene, $28.5 \%$ of the contigs had GO terms assigned, all within "Molecular function". 15\% of those is assigned to the transporter activity category [24], while this category comprises $61 \%$ in Z filipendulae. In H. melpomene, 20\% of GO annotated sequences are assigned to the "binding" category, similar to that observed in Z. filipendulae. These terms account for a larger fraction of the overall assignments than expected. This may indicate that genes encoding these functions in general are more con- served among different organisms and thus easier to annotate. Alternatively, this may represent database annotation artifacts. The "structural molecule" category is represented by $30 \%$ in $H$. melpomene, but limited to around $2 \%$ in $Z$. filipendulae. This may reflect that the original cDNA library from which the $H$. melpomene sequences were obtained was non-normalized, and therefore gave rise to an overrepresentation of ribosomal sequences. The assignments to the other categories within Molecular function are similar in $H$. melpomene and $Z$. filipendulae [24]. Differences in gene assignments between $H$. melpomene and $Z$. filipendulae could signify different selection pressures on the genes in the two species, derive from the fact that many of the Heliconius ESTs originate from wing disc cDNA libraries, and not larvae as in Z. filipendulae, or, more likely, be artifacts resulting from a low number of GO-annotated genes in both species.

In the "Biological process" group, the term representing the largest gene fraction in $Z$. filipendulae is pigmentation. While "metabolic process" and "multicellular organismal process" are relatively common terms which would be expected to feature prominently in the distribution of terms, "pigmentation" would only be expected to generate few counts among a random set of genes. Hence, the relatively high predominance of genes associated with "pigmentation" was unexpected. This may indicate that genes involved in pigmentation are conserved between insect species or more likely reflect that pigmentation is a frequently studied topic in butterflies and D. melanogaster [24]. The distribution of hits in the "Cellular component" category features organelle, cell part and organelle part as dominant terms. These are broad terms, and it seems reasonable that they are widely represented in $Z$. filipendulae. As GO and other public databases are populated with additional data, it will be possible to provide annotation for a higher fraction of the $Z$. filipendulae data set.

\section{Candidates for enzymes involved in biosynthesis of cyanogenic glucosides}

The two multigene families, P450s and UGTs, are found in most or all living organisms. They serve important roles in housekeeping reactions, as well as in the synthesis of bio-active natural products, and in detoxification of xenobiotics and allelochemicals. The pyrosequencing 
approach was therefore expected to provide a multitude of sequences within both families of which the great majority was not expected to have any function related to cyanogenesis in $Z$. filipendulae. To select genes putatively related to biosynthesis of CNglcs, the presumed high transcriptional activity of the relevant P450 and UGT encoding genes, reflecting the demand for effective de novo CNglc biosynthesis in $Z$. filipendulae larvae, was employed. Therefore, P450 and UGT gene sequences providing a high number of reads and long contigs were considered promising gene candidates. Additional priority was given to candidate genes with no close homologs in the genomes of acyanogenic B. mori and D. melanogaster, because a high sequence identity would probably signify a housekeeping protein. In cases where putative homologs were found in other sequenced insects, sufficient time may have passed since the divergence from a common ancestor for recruitment of the gene to a new function. The shared ability of $Z$. filipendulae and Heliconius species to produce the CNglcs linamarin and lotaustralin motivated a search for putative orthologous genes in Heliconius. Since the estimated time of divergence between B. mori and H. melpomene is $103 \pm 9 \mathrm{MY}$ [20], the divergence between $Z$. filipendulae and Heliconius is probably comparable (Figure 4). However, only very few genes from the Heliconius dataset matched $Z$. filipendulae genes belonging to the P450 or UGT multigene families. This is probably due to the fact that the $Z$. filipendulae transcriptome is derived from larval tissue, and Heliconius ESTs are mostly derived from wing discs. Furthermore, the Heliconius ESTs are biased as biosynthesis of CNglcs in insects in general takes place in the larval stage, and not in wing tissue.

Many of the partial gene sequences obtained by pyrosequencing of the $Z$. filipendulae transcriptome belong to the same gene. The number of gene sequences found in this study predicted to encode P450s or UGTs is therefore an overestimate of the actual number of genes belonging to each of these families in Z. filipendulae. Nevertheless, the number of gene sequences found within each gene family appears to closely match the gene numbers within these groups obtained from fully sequenced insect genomes ( $A$. aegypti, A. gambiae, A. mellifera, B. mori, C. quinquefasciatus, $D$. melanogaster, $T$. castaneum) when overestimation is taken into account. The pyrosequencing approach thus afforded a high coverage of the $Z$. filipendulae transcriptome, and that conclusion was also obtained following pyrosequencing of the transcriptomes of other organisms [42].

\section{Evolution and selection on enzymes involved in biosynthesis of cyanogenic glucosides}

Highly expressed genes are often characterized by a higher codon usage bias and low ENC numbers, in comparison to lowly expressed genes [33]. In the P450 and UGT multigene families investigated here, the codon usage bias is surprisingly low and the ENC numbers high, considering that the full length copies represent a fairly high expression level. High ENC values have previously been found in highly expressed ribosomal proteins from Spodoptera frugiperda and B. mori, but not in D. melanogaster [34], suggesting that genes with an unbiased codon usage may be a common trait in Lepidoptera. This study indicates that this observation is also valid for $Z$. filipendulae. A quantitatively equivalent level of the tRNAs corresponding to each of the codons for the same amino acid would explain the lack of codon usage bias [34].

A recent recruitment of genes related to cyanogenesis in $Z$. filipendulae was expected to be manifested by positive selection. Nevertheless, no positive selection was observed among the two gene families examined, and this would imply that the genes tested have reached a fixed and important function, that needs to be maintained. Biosynthesis of linamarin and lotaustralin was recently shown to be old traits, at least within Zygaenidae, and maybe even in the common ancestor of butterflies and moths $[43,44]$. Bouts of positive selection present upon recruitment may long since have been masked by a subsequent long period of purifying selection to maintain sequence, once the optimal sequence had been obtained. The fact that large parts of UGTs seem to undergo neutral evolution, fits with the fact that UGTs generally have low sequence conservation albeit conserved secondary and tertiary structures [40]. The $\mathrm{N}$-terminal part of the protein, responsible for binding of the aglycone, is less conserved than the C-terminal part which binds the UDP sugar. Therefore the $\mathrm{N}$-terminal part could be the part of the sequences undergoing neutral evolution.

None of the lead candidate sequences for genes involved in biosynthesis of CNglcs in Z. filipendulae show high homology to plant genes involved in CNglc biosynthesis. This supports the notion that the ability to de novo biosynthesize CNglcs was developed independently in arthropods and plants [5] by convergent evolution. However, the similarities of the biosynthetic pathways between the two kingdoms indicate that CNglcs may be produced according to an optimal biochemical route affording easy recruitment of the enzyme activities required.

\section{Conclusion}

Pyrosequencing is an attractive approach to gain access to genes in the synthesis of bio-active natural products from insects and other organisms, for which the genome sequence is not known. The transcriptome of $Z$. filipendulae offers a unique opportunity to characterize the biosynthetic pathway of cyanogenesis in insects. Moreover, Zygaena species have co-evolved with their cyanogenic 
feed plants for millions of years [44], offering opportunities for further analyses in this field.

Analysis of the $Z$. filipendulae transcriptome by 454 pyrosequencing has revealed several gene candidates for biosynthesis of CNglcs, and full length sequences of the gene candidates have been generated by RACE PCR. Heterologous expression and biochemical and functional characterization of the recombinant enzymes will serve to identify genes with relevance to cyanogenesis. The obtained transcriptome offers the additional option to design microarrays to study transcript regulation to understand the environmental cues, timing and tissue localization of biosynthesis of CNglcs. A detailed transcriptomics comparison using such microarrays as a comparison between $Z$. filipendulae larvae that are sequestering versus de novo biosynthesizing CNglcs will further the understanding of how metabolism is directed by the content and ratio of CNglcs in the host plant.

\section{Methods}

\section{Insect material, pyrosequencing and assembly}

Z. filipendulae larvae were collected from a fallow field in Kvistgård north of Copenhagen, Denmark, and reared for a week on L. corniculatus plants devoid of CNglcs [16]. The gut was removed from one larva, and total RNA was extracted from the remaining part of the body using RNeasy plant mini kit from Qiagen (Cat. No. 74904). Integrity of the RNA was verified using an Agilent 2100 Bioanalyzer before total RNA was shipped to Eurofins MWG Operon, Inc, Germany http://www.eurofinsdna.com/home.html. Eurofins MWG Operon performed mRNA purification and normalization in order to thin out the highly abundant transcripts before 454 pyrosequencing. cDNA was produced and fragmented by nebulization, and 454 pyrosequencing carried out using a GS FLX system. Sequencing of the Z. filipendulae transcriptome resulted in 319,956 reads [NCBI Short Read Archive SRX008323] which were assembled using the "mira" assembler [45] with default settings (minimum 30 bases overlap with $80 \%$ identity). A minimum read length of 30 bases was required for assembly.

\section{Genome comparison}

Contigs and singlets from the $Z$. filipendulae transcriptome were BLASTx searched [46] against the protein databases "nr" and "Uniprot" [47], and the results checked manually. Of the 29,857 Z. filipendulae contigs, 8,395 matched proteins in "Uniprot" and 10,423 matched proteins in "nr", while the numbers for the 42,215 singlets where 4,830 and 6,715 respectively. The overall statistics of the BLAST-matches are listed in Table 1 . The matches at different levels of identity and coverage, i.e. the degree of confidence to the assignment of contig or singlet to a particular protein is as presented in [17]. The $Z$. filipendulae sequences were BLASTx searched against a D. melanogaster protein set, downloaded from Flybase (December 2008) http://flybase.org[48], and against the B. mori consensus gene dataset downloaded from the silkworm website http://silkworm.genomics.org.cn (November 2008) [49]. Protein sequences from T. castaneum, A. gambiae, and A. mellifera were downloaded from http://www.beetle base.org[50], http://www.anobase.org[18], and http:// www.beebase.org[51] respectively (August 2009). The $Z$. filipendulae sequences were also compared to 22,544 ESTs from Heliconius species downloaded from NCBI's EST collection using the keyword "Heliconius". The two species represented in the data set were $H$. melpomene $(4,977$ ESTs) and H. erato (17,567 ESTs) (the H. erato set containing 9,394 ESTs from combined $H$. erato/H. himera libraries). These two data sets were assembled separately using the EST sequence assembler geneDistiller (Scheibye-Alsing $\mathrm{K}$ et al: EST assembly with genedistiller, submitted), and the resulting Heliconius contigs and singlets are available in additional files 4 and 5 respectively. The $Z$. filipendulae sequences were annotated using the Gene Ontology (GO) terms http://www.geneontology.org) [52] where possible.

\section{Candidates for enzymes involved in biosynthesis of cyanogenic glucosides}

Candidate genes from the multigene families P450s and UGTs were identified in the $Z$. filipendulae sequence dataset by BLAST searches (using BLASTx and BLASTn with default algorithm parameter settings) with selected protein sequences from the gene families from plants and insects. Gene candidates from $Z$. filipendulae were then BLAST searched (using BLASTx and BLASTn with default algorithm parameter settings) against the entire $Z$. filipendulae transcriptome to ensure exhaustive searches. The BLAST searches enabled the assembly of some genes which had initially been assigned to different conreads (33 out of approximately 300 conreads). The sequences of approximately 50 conreads fell completely within another contig. During searches in Butterflybase http://butterfly base.ice.mpg.de/[53], a few contigs from B. mori and Antheraea mylitta which belonged to the same gene were also identified. The P450 sequences ZfCYP4G47, ZfCYP4G48, ZfCYP4L17, ZfCYP6AE27, ZfCYP6CT1, CYP9A36, ZfCYP9A37, ZfCYP304F2, ZfCYP332A3, ZfCYP333B8, ZfCYP379A2, and ZfCYP379A3 were verified by Race-PCR using the SMART RACE CDNA Amplification Kit from Clontech (cat. 634914), and partial sequences were extended to full length.

\section{Analyses on candidate genes}

Sequences were aligned in MEGA4 [54,55] using CLUSTALW [56] with default settings, and refined manually. Phylogenetic Neighbor-Joining trees were also generated, and bootstrapped with 500 iterations in MEGA4. Codon 
Usage analysis was performed with CodonW version 1.3 http://codonw.sourceforge.net/, and codon bias was calculated using the "effective number of codons" (ENC) method [33]. GC content for $Z$. filipendulae full length sequences were calculated in MEGA4. Transcriptome GC content from A. gambiae, D. melanogaster, B. mori, T. castaneum, and A. mellifera are from http://www.kazusa.or.jp/ codon/, and for Heliconius it was calculated manually. Nonsynonymous to synonymous substitution rate ratios $\left(\omega=\left(d_{N} / d_{S}\right)\right)$ were calculated for full length sequences with the program codeml from the PAML package, version 4.1 [57]. Site-models 0, 1, 2, 3, 5, 7, 8 (NSsites) were tested with the settings: model $=0$, icode $=4$ (invertebrate), cleandata $=0$. The rest of the settings were default. Models were tested against each other with likelihood ratio tests [38]. Sequences from other species used for alignments and trees (Figures 6 and 7 and Additional file 2 and 3) were downloaded from Butterflybase and GenBank retaining their respective names.

\section{Authors' contributions}

$\mathrm{MZ}$ extracted gene candidates, carried out phylogenetic analyses, and drafted the manuscript. KSA did BLAST searches of the complete transcriptome and assignment of sequences to GO groups. NBJ provided RNA samples for pyrosequencing, and verified and extended P450 sequences by RACE PCR. BLM, JG and SB outlined the project, provided guidance during the work and feed-back on the manuscript. All authors read and approved the final manuscript.

\section{Additional material}

\section{Additional file 1}

Gene ontology terms and putative P450s and UGTs from the Zygaena filipendulae transcriptome. Table with the distribution of different GOterms in the categories "Molecular function", "Biological Process" and "Cellular component" for match levels 3 and better, match levels 4 and better and match level 5 and better. Tables with putative P450s and UGTs extracted from the Zygaena filipendulae 454 pyrosequencing. Click here for file

[http://www.biomedcentral.com/content/supplementary/14712164-10-574-S1.PDF]

\section{Additional file 2}

P450 tree. Neighbor-joining bootstrap tree of full length P450s from Z. filipendulae as well as P450s from other insects. Bootstrap values are shown as percentages.

Click here for file

[http://www.biomedcentral.com/content/supplementary/14712164-10-574-S2.TIFF]

\section{Additional file 3}

UGT tree. Neighbor-joining bootstrap tree of full length UGTs from Z. filipendulae as well as UGTs from other insects. Bootstrap values are shown as percentages.

Click here for file

[http://www.biomedcentral.com/content/supplementary/1471-

2164-10-574-S3.TIFF]

\section{Additional file 4}

Heliconius contigs. FASTA file of all Heliconius contigs. Click here for file

[http://www.biomedcentral.com/content/supplementary/1471-

2164-10-574-S4.PDF]

\section{Additional file 5}

Heliconius singlets. FASTA file of all Heliconius singlets. Click here for file

[http://www.biomedcentral.com/content/supplementary/14712164-10-574-S5.PDF]

\section{Acknowledgements}

We are grateful to David Nelson http://drnelson.utmem.edu/ CytochromeP450.html, and the UGT Nomenclature Committee http:// www.pharmacogenomics.pha.ulaval.ca/sgc/ugt alleles/ for naming our full length UGTs and P450s. This work was supported by the Danish research council for Technology and Production (FTP), by the grant from the Villum Kann Rasmussen Foundation to Pro-Active Plants and by Center for Applied Bioinformatics, LIFE, University of Copenhagen.

\section{References}

I. Nahrstedt A: Cyanogenesis in the Zygaenidae (Lepidoptera): a review of the state of the art. In Proceedings of the 5th International Symposium on the Biology of the Zygaenidae (Insecta, Lepidoptera) Edited by: Tremewan WG, Wipking W, Naumann CM. Grietherbusch (Germany): Koeltz Scientific books; 1993:17-29.

2. Bak S, Paquette SM, Morant M, Rasmussen AV, Saito S, Bjarnholt N, et al.: Cyanogenic glycosides; a case study for evolution and application of cytochromes P450. Phytochemistry rev 2006, 5:309-329.

3. Duffey SS: Cyanide and Arthropods. In Cyanide in Biology Edited by: Vennesland B, Conn EE, Knowles CJ, Westley J, Wissing F. London: Academic press; 1981:385-414.

4. Davis RH, Nahrstedt A: Cyanogenesis in insects. In Comprehensive Insect Physiology, Biochemistry and Pharmacology Edited by: Kerkut GA, Gilbert LI. Oxford: Pleanum Press; 1 985:635-654.

5. Zagrobelny M, Bak S, Møller BL: Cyanogenesis in plants and arthropods. Phytochemistry 2008, 69:1457-I468.

6. Bak S, Kahn RA, Nielsen HL, Møller BL, Halkier BA: Cloning of three A type cytochromes P450, CYP7IEI, CYP98, and CYP99 from Sorghum bicolor (L.) Moench by a PCR approach and identification by expression in Escherichia coli of CYP7IEI as a multifunctional cytochrome P450 in the biosynthesis of the cyanogenic glucoside dhurrin. Plant Mol Biol 1998, 36:393-405.

7. Sibbesen O, Koch B, Halkier BA, Møller BL: Isolation of the hemethiolate enzyme cytochrome $\mathrm{P450}_{\mathrm{Tyr}}$, Which catalyces the committed step in the biosynthesis of the cyanogenic glucoside dhurrin in Sorghum bicolor (L.) Moench. PNAS 1994, 91:9740-9744.

8. Jones PR, Møller BL, Høj PB: The UDP-glucose: $\mathbf{p}$-hydroxymandelonitrile-O-glucosyltransferase that catalyzes the last step in synthesis of the cyanogenic glucoside dhurrin in Sorghum bicolor - isolation, cloning, heterologous expression, and substrate specificity. J Biol Chem 1999, 274:35483-3549I. 
9. Koch B, Nielsen VS, Halkier BA, Olsen CE, Møller BL: The biosynthesis of cyanogenic glucosides in seedlings of cassava (Manihot esculenta Cranz). Arch Biochem Biophys 1992, 292: I4I-I 50.

I0. Jørgensen K, Bak S, Busk PK, Sørensen C, Olsen CE, Puonti-Kaerlas J, et al.: Cassava Plants with a Depleted Cyanogenic Glucoside Content in Leaves and Tubers. Distribution of Cyanogenic Glucosides, Their Site of Synthesis and Transport, and Blockage of the Biosynthesis by RNA Interference Technology. Plant Physiol 2005, I 39:363-374.

II. Davis RH, Nahrstedt A: Biosynthesis of cyanogenic glucosides in butterflies and moths - effective incorporation of 2-methylpropanenitrile and 2-methylbutanenitrile into linamarin and lotaustralin by Zygaena and Heliconius species (Lepidoptera). Insect Biochem 1987, I 7:689-693.

12. Holzkamp G, Nahrstedt A: Biosynthesis of cyanogenic glucosides in the Lepidoptera - Incorporation of [U-C-I 4]-2-methylpropanealdoxime, 2S-[U-C-I4]-methylbutanealdoxime and D, L-[U-C-I4]-N-hydroxyisoleucine into linamarin and lotaustralin by the larvae of Zygaena trifolii. Insect Biochem Mol Biol I994, 24:161-165

13. Wray V, Davis RH, Nahrstedt A: Biosynthesis of cyanogenic glycosides in butterflies and moths - incorporation of valine and isoleucine into linamarin and lotaustralin by Zygaena and Heliconius species (Lepidoptera). Z Naturforsch C 1983, 38:583-588.

14. Zagrobelny M, Olsen CE, Bak S, Møller BL: Intimate roles for cyanogenic glucosides in the life cycle of Zygaena filipendulae (Lepidoptera, Zygaenidae). Insect Biochem Mol Biol 2007, 37: I I89-II97.

I5. Werck-Reichhart D, Feyereisen R: Cytochromes P450: a succes story. Genome Biol 2000, I:3003. I-3003.9.

16. Zagrobelny M, Bak S, Ekstrøm CT, Olsen CE, Møller BL: The cyanogenic glucoside composition of Zygaena filipendulae (Lepidoptera: Zygaenidae) as effected by feeding on wild-type and transgenic lotus populations with variable cyanogenic glucoside profiles. Insect Biochem Mol Biol 2007, 37:10-18.

17. Gorodkin J, Cirera S, Hedegaard J, Gilchrist MJ, Panitz F, Jørgensen C, et al:: Porcine transcriptome analysis based on 97 non-normalized cDNA libraries and assembly of I,02 I,89I expressed sequence tags. Genome Biol 2007, 8:R45.

18. Holt RA, Subramanian GM, Halpern A, Sutton GG, Charlab R, Nusskern DR, et al:: The Genome Sequence of the Malaria Mosquito Anopheles gambiae. Science 2002, 298: |29-149.

19. Zdobnov EM, von Mering C, Letunic I, Torrents D, Suyama M, Copley RR, et al:: Comparative Genome and Proteome Analysis of Anopheles gambiae and Drosophila melanogaster. Science 2002, 298: $149-159$.

20. Pringle EG, Baxter SW, Webster CL, Papanicolaou A, Lee SF, Jiggins $C D$ : Synteny and Chromosome Evolution in the Lepidoptera: Evidence From Mapping Heliconius melpomene. Genetics 2007, I 77:417-426.

21. Engler HS, Spencer KC, Gilbert LE: Insect metabolism - preventing cyanide release from leaves. Nature 2000, 406:।44-|45.

22. Nahrstedt A, Davis RH: Occurrence, variation and biosynthesis of the cyanogenic glucosides linamarin and lotaustralin in species of the Heliconiini (Insecta, Lepidoptera). Comp Biochem Physiol PT B 1983, 75:65-73.

23. Jiggins $C D$, Mcmillan WO, Mallet J: Host plant adaptation has not played a role in the recent speciation of Heliconius himera and Heliconius erato. Ecol Entomol 1997, 22:361-365.

24. Papanicolaou A, Joron M, Mcmillan WO, Blaxter ML, Jiggins CD: Genomic tools and cDNA derived markers for butterflies. Mol Ecol 2005, I 4:2883-2897.

25. Paquette SM, Jensen K, Bak S: A web-based resource for the Arabidopsis P450, cytochromes b5, NADPH-cytochrome P450 reductases, and Family I Glycosyltransferases. Phytochemistry 2009, 70: 1940-1947 [http://www.p450.kvl.dk].

26. Feyereisen R: Insect Cytochrome P450. In Comprehensive Molecular Insect Science Volume 4. Edited by: Gilbert LI, latrou K, Gill SS Elsevier; 2005: I-77.

27. Feyereisen R: Evolution of insect P450. Biochem Soc Trans 2006, 34: $1252-1255$

28. Chung H, Sztal T, Pasricha S, Sridhar M, Batterham P, Daborn PJ: Characterization of Drosophila melanogaster cytochrome P450 genes. PNAS 2009. doi: 10.1073/pnas.08।2141106.
29. Vogt T, Jones P: Glycosyltransferases in plant natural product synthesis: characterization of a supergene family. Trends Plant Sci 2000, 5:380-386.

30. Jones PR, Moller BL, Hoj PB: The UDP-glucose: p-hydroxymandelonitrile-O-glucosyltransferase that catalyzes the last step in synthesis of the cyanogenic glucoside dhurrin in Sorghum bicolor - Isolation, cloning, heterologous expression, and substrate specificity. J Biol Chem 1999, 274:35483-3549I.

31. Kristensen C, Morant M, Olsen CE, Ekstrøm CT, Galbraith DW Møller BL, et al.: Metabolic engineering of dhurrin in transgenic Arabidopsis plants with marginal inadvertent effects on the metabolome and transcriptome. PNAS 2005, I 02: I779-I784.

32. Paquette SM, Møller BL, Bak S: On the origin of family I plant glycosyltransferases. Phytochemistry 2003, 62:399-4I3.

33. Wright F: The "effective number of codons" used in a gene. Gene 1990, 87:23-29.

34. Landais I, Ogliastro M, Mita K, Nohata J, López-Ferber M, DuonorCérutti M, et al:: Annotation pattern of ESTs from Spodoptera frugiperda Sf9 cells and analysis of the ribosomal protein genes reveal insect-specific features and unexpectedly low codon usage bias. Bioinformatics 2003, 19:2343-2350.

35. Savard J, Tautz D, Richards S, Weinstock GM, Gibbs RA, Werren JH, et al:: Phylogenomic analysis reveals bees and wasps (Hymenoptera) at the base of the radiation of Holometabolous insects. Genome res 2006, I6: I 334- I338.

36. Gomez SM, Eiglmeier K, Segurens B, Dehoux P, Couloux A, Scarpelli $C$, et al:: Pilot Anopheles gambiae full-length cDNA study: sequencing and initial characterization of 35,575 clones. Genome Biol 2005, 6:. doi:10.1 I86/gb-2005-6-4-r39.

37. Yang Z, Bielawski JP: Statistical methods for detecting molecular adaptation. TREE 2000, I 5:496-503.

38. Yang Z: Likelihood Ratio Tests for Detecting Positive Selection and Application to Primate Lysozyme Evolution. Mol Biol Evol 1998, I 5:568-573.

39. Osmani SA, Bak S, Imberty A, Olsen CE, Møller BL: Catalytic Key Amino Acids and UDP-Sugar Donor Specificity of a Plant Glucuronosyltransferase, UGT94B I: Molecular Modeling Substantiated by Site-Specific Mutagenesis and Biochemical Analyses. Plant Physiol 2008, I48:|295-|308.

40. Osmani S, Bak S, Møller BL: Substrate specificity of plant UDP. dependent glycosyltransferases predicted from crystal structures and homology modeling. Phytochemistry 2009, 70:325-247.

4I. Zou Z, Najar F, Wang Y, Roe B, Jiang H: Pyrosequence analysis of expressed sequence tags for Manduca sexta haemolymph proteins involved in immune responses. Insect Biochem $\mathrm{Mol} B i o l$ 2008, 38:677-682.

42. Weber APM, Weber KL, Carr K, Wilkerson C, Ohlrogge JB: Sampling the Arabidopsis Transcriptome with Massively Parallel Pyrosequencing. Plant Physiol 2007, 144:32-42.

43. Zagrobelny $M$, Bak S, Rasmussen AV, Jørgensen B, Naumann CM, Møller BL: Cyanogenic glucosides and plant-insect interactions. Phytochemistry 2004, 65:293-306.

44. Niehuis O, Naumann CM, Misof B: Phylogenetic analysis of Zygaenoidea small-subunit rRNA structural variation implies initial oligophagy on cyanogenic host plants in larvae of the moth genus Zygaena (Insecta:Lepidoptera). Zool J Linn soc 2006, |47:367-38|.

45. Chevreux B, Pfisterer T, Drescher B, Driesel AJ, Mller WEG, Wetter $\mathrm{T}$, et al:: Using the miraEST Assembler for Reliable and Automated mRNA Transcript Assembly and SNP Detection in Sequenced ESTs. Genome Res 2004, I4: I |47- I I 59.

46. Altschul SF, Gish W, Miller W, Myers EW, Lipman DJ: Basic local alignment search tool. J Mol Biol 1990, 21 5:403-4I0.

47. The UniProt Consortium: The Universal Protein Resource (UniProt). NAR 2008, 36:DI90-DI95

48. Tweedie S, Ashburner M, Falls K, Leyland P, McQuilton P, Marygold $S$, et al: FlyBase: enhancing Drosophila Gene Ontology annotations. NAR 2009, 37:D555-D559.

49. Wang J, Xia Q, He X, Dai M, Ruan J, Chen J, et al:: SilkDB: a knowledgebase for silkworm biologyand genomics. NAR 2005, 33:D399-D402.

50. Tribolium Genome Sequencing Consortium: The genome of the model beetle and pest Tribolium castaneum. Nature 2008 , 452:949-955. 
51. The Honeybee Genome Sequencing Consortium: Insights into social insects from the genome of the honeybee Apis mellifera. Nature 2006, 443:931-949.

52. The Gene Ontology Consortium: Gene ontology: tool for the unification of biology. Nat Genet 2000, 25:25-29.

53. Papanicolaou A, Gebauer-Jung S, Blaxter ML, Mcmillan WO, Jiggins $C D$ : Butterflybase: a platform for lepidopteran genomics. NAR 2008, 36:D582-D587.

54. Tamura K, Dudley J, Nei M, Kumar S: MEGA4: Molecular Evolutionary Genetics Analysis (MEGA) Software Version 4.0. Mol Biol Evol 2007, 24:1596-1599.

55. Kumar S, Nei M, Dudley J, Tamura K: MEGA: A biologist-centric software for evolutionary analysis of DNA and protein sequences. Briefings in Bioinformatics 2008, 9:299-306.

56. Larkin MA, Blackshields G, Brown NP, Chenna R, McGettigan PA, McWilliam H, et al:: Clustal $\mathbf{W}$ and Clustal $\mathbf{X}$ version 2.0. Bioinformatics 2007, 23:2947-2948.

57. Yang Z: PAML 4: Phylogenetic Analysis by Maximum Likelihood. Mol Biol Evol 2007, 24:1586-1591.

Publish with Bio Med Central and every scientist can read your work free of charge

"BioMed Central will be the most significant development for disseminating the results of biomedical research in our lifetime. "

Sir Paul Nurse, Cancer Research UK

Your research papers will be:

- available free of charge to the entire biomedical community

- peer reviewed and published immediately upon acceptance

- cited in PubMed and archived on PubMed Central

- yours - you keep the copyright

Submit your manuscript here:

http://www.biomedcentral.com/info/publishing_adv.asp
BioMedcentral 